\title{
Different Analytical Techniques, Pretreatment Methods and Adsorbent Materials for the Determination of Trans, Trans-Muconic Acid in Urine Samples
}

\author{
Xiaoyue Shan, Bingsheng Yang, Yanpeng Shi, Siwei Tan, Ji Shao, Kewen Su, Ling Zhang and \\ Haipeng Ye* \\ Hangzhou Occupational Disease Prevention and Control Hospital, Hangzhou 310014, China \\ *Corresponding author e-mail: 617304832@qq.com
}

Keywords: Urine, trans-gluconic acid, adsorbent.

\begin{abstract}
Benzene is a human carcinogen and its metabolite, Trans, trans-muconic acid (tt-MA) has been introduced by some environmental and occupational health regulatory associations as a biological index for the assessment of benzene exposure. This paper summaried and compared different analytical techniques, pretreatment methods and adsorbent materials for the determination of tt-MA in urine samples.
\end{abstract}

\section{Introduction}

Benzene is a major industrial chemical that has been used in numerous production and synthesis processes in the petrochemical, paint, plastic, detergents, drugs, rubber and dye industries [1-3]. According to the International Agency for Research on Cancer (IARC), benzene is classified as a group I human carcinogen [4, 5]. Long-term occupational and environmental exposure to benzene may cause bone marrow damage, leukemia, aplastic anemia and so on [6]. Trans, trans-muconic acid (tt-MA) and S-phenylmercapturic acid (S-PMA) are the most commonly used urinary biomarkers associated with benzene exposure in humans to be detected from urine or blood [7-9]. The American Conference of Governmental Industrial Hygienists (ACGIH) has recommended tt-MA as a biological exposure index (BEI) which is suitable urinary biomarker of benzene at high concentrations (above 1 ppm) [1, 10-12]. It appears that tt-MA is a more specific biomarker than other metabolites at high levels of benzene exposure.

Several analytical methods, including high-performance liquid chromatography (HPLC), gas chromatography (GC), ultraviolet and visible spectrophotometry (UV-vis) and mass spectrometry (MS) have been applied for the determination of tt-MA in urine [6, 12-17]. However, owing to the low levels of tt-MA in urine samples and the interferences in complex matrixes, extraction and preconcentration processes prior to detection is necessary $[18,19]$. Solid-phase extraction (SPE) and liquid- liquid extraction (LLE) are the most widely-used sample pretreatment methods for extracting and enriching tt-MA from urine samples [20]. It is obvious the choice of appropriate adsorbent materials is a key factor for effective extraction of tt-MA. Recently, there are several types of adsorbents for SPE including metal-organic framework [12], molecularly imprinted polymers [21] and other special adsorbents.

This paper summaried and compared different analytical techniques, pretreatment methods and adsorbent materials for the determination of tt-MA in urine samples.

\section{Different analytical techniques for the determination of tt-MA in urine samples}

Various analytical approaches have been introduced to measure tt-MA in urine samples, including high-performance liquid chromatography (HPLC), gas chromatography (GC), ultraviolet and visible spectrophotometry (UV-vis) and mass spectrometry (MS). Bhandari et al. [7] developed an ultra-performance liquid chromatography-tandem mass spectrometry (UPLC-MS/MS) method for simultaneously measuring 8 biomarkers of volatile organic compound (VOC) exposure, including 
tt-MA. Ruppert et al. [14] developed a sensitive and specific method for the determination of tt-MA in urine analyzed by gas chromatography-mass spectrometry (GC-MS). Marchese et al. [15] demonstrated the use of a liquid chromatography/hybrid quadrupole time-of-flight (QqTOF) mass spectrometry instrument for simultaneous determination of urinary metabolites of benzene, toluene, xylene and styrene (the metabolites selected including tt-MA). Tranfo et al. [16] presented a HPLC/MS/MS method for reliable quantitative determination of t,t-MA. Olmos et al. [22] described a specific and precise high-performance liquid chromatography (HPLC) method for the quantification of tt-MA in human urine. Lee et al. [23] described a HPLC-DAD method for tt-MA determination with enhanced selectivity and sensitivity. As shown in Table 1, tt-MA was usually determined by HPLC methods with detection by either UV or, more recently, electrospray tandem mass spectrometry and the detection limits were found to be very low.

Table 1. Different analytical techniques for the determination of tt-MA in urine samples.

\begin{tabular}{|c|c|c|c|c|}
\hline Analyte & Linearity ranges & Techniques & LODs & References \\
\hline \multirow{4}{*}{$t$} & $0.759-759 \mathrm{ng} / \mathrm{mL}$ & UPLC-MS/MS & $1.20 \mathrm{ng} / \mathrm{mL}$ & {$[7]$} \\
\cline { 2 - 5 } & $0-200 \mu \mathrm{g} / \mathrm{mL}$ & Luminescence spectra & $0.1 \mu \mathrm{mL}$ & {$[12]$} \\
\cline { 2 - 5 } & $0.01-1.00 \mu \mathrm{g} / \mathrm{mL}$ & GC-MS & $0.01 \mu \mathrm{gg} / \mathrm{mL}$ & {$[14]$} \\
\cline { 2 - 5 } & $5-1000 \mathrm{ng} / \mathrm{mL}$ & HPLC-MS/MS & $1.0 \mathrm{ng} / \mathrm{mL}$ & {$[15]$} \\
\cline { 2 - 5 } & $20-1000 \mathrm{ng} / \mathrm{mL}$ & HPLC-MS/MS & $0.22 \mathrm{ng} / \mathrm{mL}$ & {$[16]$} \\
\cline { 2 - 5 } & $10-2000 \mathrm{ng} / \mathrm{mL}$ & HPLC-UV & $10.8 \mathrm{ng} / \mathrm{mL}$ & {$[22]$} \\
\cline { 2 - 5 } & $5-5000 \mathrm{ng} / \mathrm{mL}$ & HPLC-DAD & $5.0 \mathrm{ng} / \mathrm{mL}$ & {$[23]$} \\
\hline
\end{tabular}

\section{Different pretreatment methods for the determination of tt-MA in urine samples}

The direct analysis of tt-MA in urine is not recommended owing to the low levels of tt-MA and the complexity of biological matrixes. Therefore, extraction and preconcentration steps in the analytical procedure is necessary to enhance the sensitivity and selectivity. Bahrami et al. [19] developed a novel technique for extraction and determination of trans,trans-muconic acid (tt-MA), hippuric acid (HA) and mandelic acid (MA) by means of ion-pair-based hollow fiber liquid-phase microextraction (IP-HF-LPME). Mudiam et al. [24] reported a MIP using methacrylic acid (MAA) as a monomer followed by simultaneous derivatization using ethyl chloroformate and pre-concentration using dispersive liquid-liquid microextraction (DLLME) for the determination of tt-MA in urine samples. Gomes et al. [25] developed an analytical method for the determination of urinary tt-MA using solid phase extraction (SPE) onto a Strata SAX cartridge. Rismanchian et al. [26] detected tt-MA in urine samples using partitioned dispersive liquid-liquid microextraction technique (PDLLME) coupled with high-performance liquid chromatography-UV detection. Chauhan et al. [27] synthesized a molecularly imprinted polymer (MIP) and used it as an adsorbent for solid phase extraction of six urinary benzene metabolites (UBMs). Table 2 clearly demonstrates that SPE and LLE are the most widely-used sample pretreatment methods in the analysis of tt-MA in urine samples.

Table 2. Different pretreatment methods for the determination of tt-MA in urine samples.

\begin{tabular}{|c|c|c|c|c|}
\hline Analyte & Sample pretreatment methods & Techniques & LODs & References \\
\hline \multirow{4}{*}{ tt-MA } & IP-HF-LPME & HPLC-UV & $0.0001 \mu \mathrm{g} / \mathrm{mL}$ & {$[19]$} \\
\cline { 2 - 5 } & SPE & HPLC-UV & $5.0 \mu \mathrm{g} / \mathrm{L}$ & {$[23]$} \\
\cline { 2 - 5 } & MIP-DLLME & GC-MS & $0.037 \mu \mathrm{g} / \mathrm{mL}$ & {$[24]$} \\
\cline { 2 - 5 } & SPE & HPLC-UV & $0.11 \mathrm{ng} / \mathrm{mL}$ & {$[25]$} \\
\cline { 2 - 5 } & PDLLME & GPC-UV & $0.11 \mathrm{ng} / \mathrm{mL}$ & {$[26]$} \\
\cline { 2 - 5 } & MISPE & $1.2 \mathrm{ng} / \mathrm{mL}$ & {$[27]$} \\
\hline
\end{tabular}




\section{Different adsorbent materials for the determination of tt-MA in urine samples}

Due to the key role of the adsorbents in SPE technique, new types of SPE adsorbents to improve the extraction efficiency for tt-MA were explored. Qu et al. [12] synthesized a luminescent lanthanide-based MOFs hybrid material (Tb(III)@MOF-SO3) as a fluorescence sensor based on fluorescence quenching mechanisms to detect tt-MA in a urine system. Soleimani et al. [21] developed a novel approach based on a molecularly imprinted polymer (MIP) in microextraction by packed sorbent (MEPS), followed by high-performance liquid chromatography with ultra-violet detection (MIMEPS-HPLC-UV) to determine tt-MA in urine samples. Vieira et al. [28] synthesized a MIP (using 4-vinylpyridine as functional monomer) based on molecularly imprinted solid-phase extraction (MISPE) for the extraction of tt-MA in urine samples, followed by high-performance liquid chromatography with ultraviolet detection (HPLC-UV). Mudiam et al. [24] synthesized a MIP (using methacrylic acid as functional monomer) followed by simultaneous ethyl chloroformate derivatization and preconcentration by dispersive liquid-liquid microextraction (DLLME) for the analysis of tt-MA in urine samples using gas chromatography-mass spectrometry (GC-MS), as shown in Table 3.

Table 3. Different adsorbent materials for the determination of tt-MA in urine samples.

\begin{tabular}{|c|c|c|c|c|c|}
\hline Analyte & Adsorbent materials & $\begin{array}{c}\text { Sample preparation } \\
\text { techniques }\end{array}$ & Techniques & LODs & References \\
\hline \multirow{4}{*}{ tt-MA } & Tb(III)@MOF-SO ${ }_{3}$ & $/$ & $\begin{array}{c}\text { Luminescence } \\
\text { spectra }\end{array}$ & $0.1 \mu \mathrm{g} / \mathrm{mL}$ & {$[12]$} \\
\cline { 2 - 6 } & MIP & MIMEPS & HPLC-UV & $0.015 \mu \mathrm{g} / \mathrm{mL}$ & {$[21]$} \\
\cline { 2 - 6 } & MIP & MISPE & HPLC-UV & $0.3 \mu \mathrm{mL}$ & {$[28]$} \\
\cline { 2 - 6 } & MIP & DLLME & GC-MS & $0.037 \mu \mathrm{g} / \mathrm{mL}$ & {$[24]$} \\
\hline
\end{tabular}

\section{Conclusion}

Many methods have been applied to determine tt-MA in urine samples, including HPLC-UV, GC-MS, luminescence spectra and ect. Among them, HPLC-UV is the most widely-used technique. Due to the complexity of real urine sample matrix, SPE and LLE are frequently applied as sample pretreatment methods. And new types of SPE adsorbent materials are developed to improve the extraction efficiency for tt-MA determination.

\section{Acknowledgments}

This work was financially supported by the Hangzhou Foundation for Development of Science and Technology (NO. 20180533B95, NO. 20181228Y28), the Medical Health Foundation for Key Talents in Zhejiang Province, China (NO. 2016KYB241, NO. 2018KY640, NO. 2019KY543).

\section{References}

[1] M. Carrieri, E. Bonfiglio, M.L. Scapellato, I. Macca, G. Tranfo, P. Faranda, E. Paci, G.B. Bartolucci, Comparison of exposure assessment methods in occupational exposure to benzene in gasoline filling-station attendants, Toxicology letters. 162 (2006) 146-52.

[2] A. Jalai, Z. Ramezani, K. Ebrahim, Urinary Trans, Trans-Muconic Acid is Not a Reliable Biomarker for Low-level Environmental and Occupational Benzene Exposures, Safety and health at work. 8 (2017) 220-225.

[3] S.L.C. Daniele Merli, Federica Balduzzi, Ana Miljkovic, Lucio Toma and Valeria Amendola, A smart supramolecular device for the detection of t,t-muconic acid in urine, New J. Chem. 42 (2018) 15460-15465 
[4] Y.Z. Baghdady K.A. Schug, A novel diagnostic in situ derivatization kit for the simultaneous determination of 14 biomarkers of exposure to benzene, toluene, ethyl benzene and xylenes in human urine by isotope dilution liquid chromatography tandem mass spectrometry and kit optimization using response surface methodology, Analytica chimica acta. 1036 (2018) 195-203.

[5] A. Barbieri, L. Sabatini, A. Accorsi, A. Roda, F.S. Violante, Simultaneous determination of t,t-muconic, S-phenylmercapturic and S-benzylmercapturic acids in urine by a rapid and sensitive liquid chromatography/electrospray tandem mass spectrometry method, Rapid communications in mass spectrometry : RCM. 18 (2004) 1983-8.

[6] P. Manini, G. De Palma, R. Andreoli, D. Poli, P. Mozzoni, G. Folesani, A. Mutti, P. Apostoli, Environmental and biological monitoring of benzene exposure in a cohort of Italian taxi drivers, Toxicology letters. 167 (2006) 142-51.

[7] D. Bhandari, D. McCarthy, C. Biren, C. Movassaghi, B.C. Blount, V.R. De Jesus, Development of a UPLC-ESI-MS/MS method to measure urinary metabolites of selected VOCs: Benzene, cyanide, furfural, furfuryl alcohol, 5-hydroxymethylfurfural, and N-methyl-2-pyrrolidone, Journal of chromatography. B, Analytical technologies in the biomedical and life sciences. 1126-1127 (2019) 121746.

[8] S.F. Belal, R.A. Shaalan, M.A.A. Ragab, E.A. El-Attar, M. Agami, Liquid Chromatography-Mass Spectrometry for muconic, mandelic, hippuric and methylhippuric acids analysis in human urine as metabolites for fuel exposure, Microchemical Journal. 149 (2019) 103992.

[9] S. Bukkawar, N. Sarwade, M. Panse, Polyaniline assisted USB based sensor for determination of benzene biomarker, Sensing and Bio-Sensing Research. 22 (2019) 100260.

[10] F. Ghamari, A. Bahrami, Y. Yamini, F.G. Shahna, A. Moghimbeigi, Development of Hollow-Fiber Liquid-Phase Microextraction Method for Determination of Urinary trans,trans-Muconic Acid as a Biomarker of Benzene Exposure, Analytical chemistry insights. 11 (2016) 65-71.

[11] M. Carrieri, G. Spatari, G. Tranfo, D. Sapienza, M.L. Scapellato, G.B. Bartolucci, M. Manno, Biological monitoring of low level exposure to benzene in an oil refinery: Effect of modulating factors, Toxicology letters. 298 (2018) 70-75.

[12] X.L. Qu B. Yan, Ln(III)-Functionalized Metal-Organic Frameworks Hybrid System: Luminescence Properties and Sensor for trans, trans-Muconic Acid as a Biomarker of Benzene, Inorganic chemistry. 57 (2018) 7815-7824.

[13] S.J. Shahtaheri, F. Ghamari, F. Golbabaei, Sample preparation followed by high performance liquid chromatographic (HPLC) analysis for monitoring muconic acid as a biomarker of occupational exposure to benzene, International journal of occupational safety and ergonomics : JOSE. 11 (2005) 377-88.

[14] G.S. Thomas Ruppert , Anthony R. Tricker , Dankwart Rauscher , Franz Adlkofer Determination of urinary trans,trans-muconic acid by gas chromatography-mass spectrometry, Journal of Chromatography B. 666 (1995) 71-76.

[15] S. Marchese, R. Curini, A. Gentili, D. Perret, L.M. Rocca, Simultaneous determination of the urinary metabolites of benzene, toluene, xylene and styrene using high-performance liquid chromatography/hybrid quadrupole time-of-flight mass spectrometry, Rapid communications in mass spectrometry : RCM. 18 (2004) 265-72.

[16] G. Tranfo, E. Paci, R. Sisto, D. Pigini, Validation of an HPLC/MS/MS method with isotopic dilution for quantitative determination of trans,trans-muconic acid in urine samples of workers 
exposed to low benzene concentrations, Journal of chromatography. B, Analytical technologies in the biomedical and life sciences. 867 (2008) 26-31.

[17] W.C. Chiang, C.Y. Chen, T.C. Lee, H.L. Lee, Y.W. Lin, Fast and simple screening for the simultaneous analysis of seven metabolites derived from five volatile organic compounds in human urine using on-line solid-phase extraction coupled with liquid chromatography-tandem mass spectrometry, Talanta. 132 (2015) 469-78.

[18] A. Ostovan, M. Ghaedi, M. Arabi, A. Asfaram, Hollow porous molecularly imprinted polymer for highly selective clean-up followed by influential preconcentration of ultra-trace glibenclamide from bio-fluid, Journal of chromatography. A. 1520 (2017) 65-74.

[19] A. Bahrami, F. Ghamari, Y. Yamini, F. Ghorbani Shahna, A. Koolivand, Ion-pair-based hollow-fiber liquid-phase microextraction combined with high-performance liquid chromatography for the simultaneous determination of urinary benzene, toluene, and styrene metabolites, Journal of separation science. 41 (2018) 501-508.

[20] Z. Lin, Q. He, L. Wang, X. Wang, Q. Dong, C. Huang, Preparation of magnetic multi-functional molecularly imprinted polymer beads for determining environmental estrogens in water samples, Journal of hazardous materials. 252-253 (2013) 57-63.

[21] E. Soleimani, A. Bahrami, A. Afkhami, F.G. Shahna, Determination of urinary trans,trans-muconic acid using molecularly imprinted polymer in microextraction by packed sorbent followed by liquid chromatography with ultraviolet detection, Journal of chromatography. B, Analytical technologies in the biomedical and life sciences. 1061-1062 (2017) 65-71.

[22] S.C.t. Valentina Olmos, Clara M. L6pez, and Edda C. Villaamil, High-Performance Liquid Chromatography Method for Urinary trans, trans.Muconic Acid. Application to Environmental Exposure to Benzene, Journal of Analytical Toxicology. 30 (2006) 258-261.

[23] B.L. Lee, H.Y. Ong, Y.B. Ong, C.N. Ong, A sensitive liquid chromatographic method for the spectrophotometric determination of urinary trans,trans-muconic acid, Journal of chromatography. B, Analytical technologies in the biomedical and life sciences. 818 (2005) 277-83.

[24] M.K. Mudiam, A. Chauhan, K.P. Singh, S.K. Gupta, R. Jain, R. Ch, R.C. Murthy, Determination of t,t-muconic acid in urine samples using a molecular imprinted polymer combined with simultaneous ethyl chloroformate derivatization and pre-concentration by dispersive liquid-liquid microextraction, Analytical and bioanalytical chemistry. 405 (2013) 341-9.

[25] R. Gomes, A. Sanson, F. Lobo, R. Afonso, M. Coutrim, Method for the Determination of Benzene Metabolite t,t-Muconic Acid in Urine by HPLC-UV with an Ion Exclusion Column, Separations. 3 (2016) 14.

[26] M. Rismanchian, K. Ebrahim, Z. Ordudari, Development of a simple and rapid method for determination of trans, trans-Muconic Acid in human urine using PDLLME preconcentration and HPLC-UV detection, Chemical Papers. 73 (2019) 2485-2492.

[27] A. Chauhan, T. Bhatia, M.K. Gupta, P. Pandey, V. Pandey, P.N. Saxena, M.K. Mudiam, Imprinted nanospheres based on precipitation polymerization for the simultaneous extraction of six urinary benzene metabolites from urine followed by injector port silylation and gas chromatography-tandem mass spectrometric analysis, Journal of chromatography. B, Analytical technologies in the biomedical and life sciences. 1001 (2015) 66-74.

[28] A.C. Vieira, R.A. Zampieri, M.E. de Siqueira, I. Martins, E.C. Figueiredo, Molecularly imprinted solid-phase extraction and high-performance liquid chromatography with ultraviolet detection for the determination of urinary trans,trans-muconic acid: a comparison with ionic exchange extraction, The Analyst. 137 (2012) 2462-9. 